\title{
The Prognostic Importance of EGFR and COX-2 Expression in Cervix Cancer Stages IIb-IVa
}

\author{
Parvin Adimi $^{1}$, Karina Dahl Steffensen ${ }^{1,2}$, Doris Schledermann ${ }^{3}$, Eva Rye Rasmussen ${ }^{1}$, \\ Anders Jakobsen ${ }^{1,2}$
}

${ }^{1}$ Department of Oncology, Vejle Hospital, Vejle, Denmark; ${ }^{2}$ Institute of Regional Health Services Research, University of Southern Denmark, Odense, Denmark; ${ }^{3}$ Department of Pathology, Odense University Hospital, Odense, Denmark.

Email: anders.jakobsen@slb.regionsyddanmark.dk

Received December $3^{\text {rd }}, 2010$; revised February $2^{\text {nd }}, 2011$; accepted February $28^{\text {th }}, 2011$.

\begin{abstract}
Objectives: Locally advanced cervix cancer represents a therapeutic challenge with a delicate balance between effect and toxicity. Consequently, there is an obvious need for new prognostic parameters with a perspective of a more individualized treatment. The aim of the present study was to evaluate the possible prognostic importance of epidermal growth factor receptor and cyclooxygenase-2 expression in locally advanced cervix cancer. Methods: The study included 91 patients with cervix cancer, FIGO stages IIb-IVa, who were treated with curative intent according to the Nordic Cervix Cancer protocol (NOCECA). The median observation time was 7 years. Epidermal growth factor receptor and cyclooxygenase-2 expression was evaluated by immunohistochemistry using commercially available antibodies. The tumor marker expression was evaluated according to a semi-quantitative scoring system with a positive score when more than $10 \%$ of the tumor cells were moderately or strongly stained. Results: Epidermal growth factor receptor and cyclooxygenase-2 over-expression was found in $22 \%$ and $18 \%$ of the patients, respectively. The survival differed according to epidermal growth factor receptor and cyclooxygenase-2 over-expression as calculated by Kaplan-Meier plots and log-rank test ( $p=0.0002$ and $p=0.0084$ respectively). A multivariate Cox Regression analysis identified each tumor marker as an independent prognostic factor. Conclusion: The results clearly indicate that epidermal growth factor receptor and cyclooxygenase-2 hold important prognostic information, markedly appearing with a long-term observation. We found that both parameters were independent prognostic factors, and to further clarify this matter our retrospective analysis should be confirmed in a prospective study with more patients.
\end{abstract}

Keywords: Gynecologic Oncology, Cervical Cancer, EGFR, COX-2, Prognostic Marker

\section{Introduction}

The treatment of locally advanced (stages IIb-IVa) cervix cancer remains a major problem in gynaecological oncology. Radiotherapy is still a cornerstone in the treatment although improvements have been obtained with the introduction of concomitant chemotherapy. Progress, however, is more moderate [1] than expected from the first results 10 years ago. A major fraction of the patients cannot be cured with the present treatment options and new modalities are eagerly awaited. Introduction of new treatments should be evidence based and there is an obvious need for a better selection of patients not cured by the present treatment offered. New prognostic markers may serve that purpose and studies along that line are certainly justified.
The epidermal growth factor (EGF) system consists of several ligands and a family of four receptors. The receptors have tyrosine kinase activity, and binding of the ligands results in hetero- or homo-dimerization and phosphorylation of the intracellular domain. The activation results in a cascade of reactions ending up with cell proliferation and decreased apoptosis. There seems to be a relation between the EGF system and the vascular endothelial growth factor (VEGF) system, but the possible mutual influence needs further elucidation. The Epidermal Growth Factor Receptor (EGFR, ErbB-1) is overexpressed in several malignant tumors including cervix cancer [2]. The overexpression may appear early in the carcinogenesis and increase during the malignant development [3].

Cyclooxygenase-2 (COX-2) is a cytokine inducible 
enzyme converting arachidonic acid into prostaglandins, which have a radioprotective effect [4]. Overexpression of COX-2 may reduce the radiosensitivity and consequently signalize a poor prognosis in patients treated with radiotherapy. Furthermore, COX-2 is linked with angiogenesis [5], which may further increase the prognostic influence.

The objective of the present study was to investigate the prognostic importance of EGFR and COX-2 expression in a homogenously classified and treated patient material with stages IIb-IVa cervix cancer and long-term observation.

\section{Materials and Methods}

The study included 91 patients with cervix cancer stages IIb-IVa. All patients had histopathological verification of the diagnosis by examination of biopsy specimens. The patients were examined under general anesthesia and further classified according to FIGO guidelines. The treatment was a combination of external radiation and brachytherapy modified according to tumor size. The external treatment was based on a conformal technique given by a linear accelerator with an energy $\geq 6 \mathrm{MV}$. A 4-field technique with two A/P-P/A fields and two lateral fields was used to differentiate the dose to tumor and lymph nodes at risk. The A/P-P/A fields included tumor and pelvic lymph nodes. The daily dose was $1.8 \mathrm{~Gy}$. The daily tumor dose was increased by the two lateral fields to $2.0 \mathrm{~Gy}$ by a concomitant boost of $0.2 \mathrm{~Gy}$. Tumors 4-8 $\mathrm{cm}$ received $50 \mathrm{~Gy} / 25$ fractions and a lymph node dose of $45 \mathrm{~Gy} / 25$ fractions. The tumor dose was raised by 6 intracavitary insertions with a total dose to point $\mathrm{A}$ of $28.44 \mathrm{~Gy}$. Tumors $>8 \mathrm{~cm}$ received 60 Gy by external radiation and $20 \mathrm{~Gy} / 5$ insertions by intracavitary treatment.

\subsection{Immunohistochemistry}

Tumor specimens were obtained before any cancer therapy. For immunohistochemistry $4 \mu \mathrm{m}$ sections were cut from neutral-buffered formaldehyde-fixed paraffin-embedded tissue blocks. Sections were mounted at SuperFrost Plus slides, dried at $60^{\circ} \mathrm{C}$, deparaffinized and hydrated. Prior to antigen retrieval, blocking of endogenous peroxidase in $1.5 \%$ hydrogen peroxide in TBS buffer, $\mathrm{pH}$ 7.4 was done for $10 \mathrm{~min}$. Antigen retrieval was performed using microwave heating in $10 \mathrm{mM}$ Tris with 0.5 mM EDTA at pH 9.0 (TEG) for $11 \mathrm{~min}$ at full power (900W), then for $15 \mathrm{~min}$. at $400 \mathrm{~W}$. After heating, slides remained in the buffer for $15 \mathrm{~min}$. Incubation with antibodies (EGFR.113, dilution 1:50 (NovoCastra, Newcastle upon Tyne, UK) and $C O X-2, C X-294$, dilution 1:50 (Dako, Glostrup, Denmark) was done for $60 \mathrm{~min}$. at room temperature. Immunostaining was automated using the EnVision+TM detection system for COX-2 and PowerVision+TM detection system for EGFR.113 on the AutoStainer instrument (Dako, Glostrup, Denmark). As substrate-chromogen system DAB + K3468 was used (Dako, Glostrup, Denmark). Immunostaining was followed by brief nuclear counter staining in Mayer's haematoxylin. Finally, cover slips were mounted with AquaTex (Merck, Darmstadt, Germany).

\subsection{Evaluation of Immunohistochemic Staining}

A semi-quantitative approach was used to analyze the EGFR staining reaction. The proportion of positive tumor cells in each sample was scored as 0\%, 1 - 9\%, 10 $50 \%$ and $>50 \%$. The staining intensity was scored as no staining, low intensity, moderate intensity or strong intensity. Specimens with membranous staining at moderate or intense staining intensity in more than $10 \%$ of the tumor area were scored as positive.

The staining pattern of COX-2 was classified as score 0 when negative or unspecific, score 1 if the staining was weak and incomplete in more than $10 \%$ of tumor cells, score 2 if moderate and complete staining was detectable in more than $10 \%$ of the tumor cells, and score 3 if more than $10 \%$ of the tumor cells displayed strong and complete membranous or cytoplasmatic staining. COX-2 was scored as positive when more than $10 \%$ of the tumor area stained with a moderate or strong intensity. Very similar methods are used in several other studies [6-9].

\subsection{Statistical Analyses}

For the assessment of a correlation between markers (EGFR and COX-2) and clinicopathological parameters, chi-square statistics were employed. Univariate overall survival analysis was performed using the Kaplan-Meier method and log-rank statistics for comparison of survival curves. Multivariate overall survival analysis was based on the Cox regression model. Clinical parameters, FIGO stage and age were analyzed as continuous variables and EGFR and COX-2 as categorical variables. Statistical significance was defined as a probability level $<0.05$. All statistical analysis were done using the NCSS software (version 07.1.5, 2007, Kaysville, Utah, www.ncss.com)

\section{Results}

The patients were included from 1994 - 1999. Table 1 shows the patient characteristics. It appears that $>90 \%$ of the patients had stage IIb or IIIb and more than $90 \%$ had squamous cell carcinoma.

The Hb level was within normal range in most patients. The median follow-up time was 12 years.

Twenty cervical cancer tissue samples (22\%) expressed membranous EGFR staining at a moderate to strong intensity in more than $10 \%$ of the tumor cells and scored as 
Table 1. Patient characteristics, biomarkers and survival outcome.

\begin{tabular}{|c|c|c|c|}
\hline & Characteristics & $\mathrm{N}$ & $\%$ \\
\hline \multicolumn{2}{|l|}{ Age } & \multirow[t]{3}{*}{91} & \multirow[t]{3}{*}{100} \\
\hline Median & \multirow{2}{*}{$\begin{array}{l}63.9 \\
32.6-79.4\end{array}$} & & \\
\hline Range & & & \\
\hline \multicolumn{4}{|l|}{ FIGO stage } \\
\hline \multicolumn{2}{|l|}{ IIb } & 37 & 40.7 \\
\hline \multicolumn{2}{|l|}{ IIIa } & 1 & 1.1 \\
\hline \multicolumn{2}{|l|}{ IIIb } & 46 & 50.6 \\
\hline \multicolumn{2}{|l|}{ IV } & 7 & 7.7 \\
\hline \multicolumn{4}{|l|}{ Histology } \\
\hline \multicolumn{2}{|c|}{ Squamous } & 86 & 94.5 \\
\hline \multicolumn{2}{|c|}{ Adenocarcinoma } & 5 & 5.5 \\
\hline \multicolumn{4}{|c|}{ Pretreatment hemoglobin } \\
\hline \multicolumn{2}{|c|}{$<7.0 \mathrm{mmol} / \mathrm{L}$} & 19 & 20.9 \\
\hline \multicolumn{2}{|c|}{$\geq 7.0 \mathrm{mmol} / \mathrm{L}$} & \multirow[t]{3}{*}{72} & \multirow[t]{3}{*}{79.1} \\
\hline Median & $7.8 \mathrm{mmol} / \mathrm{L}$ & & \\
\hline Range & $5.1-9.4$ mmol/L ) & & \\
\hline \multicolumn{4}{|c|}{ EGFR immunohistochemistry } \\
\hline \multicolumn{2}{|c|}{ EGFR negative } & 71 & 78.0 \\
\hline \multicolumn{2}{|c|}{ EGFR positive } & 20 & 22.0 \\
\hline \multicolumn{4}{|c|}{ COX2 immunohistochemistry } \\
\hline \multicolumn{2}{|c|}{ COX-2 negative } & 75 & 82.4 \\
\hline \multicolumn{2}{|c|}{ COX-2 positive } & 16 & 17.6 \\
\hline \multicolumn{4}{|c|}{ Combined EGFR/COX-2 immunohistochemistry } \\
\hline \multicolumn{2}{|c|}{ EGFR and COX-2 negative } & 59 & 64.8 \\
\hline \multicolumn{2}{|c|}{ EGFR or COX-2 positive } & 32 & 35.2 \\
\hline \multicolumn{2}{|l|}{ Follow-up } & 91 & 100 \\
\hline Median & 12 & & \\
\hline Range & $8.3-14.3$ & & \\
\hline
\end{tabular}

positive, while 71 samples (78\%) were scored as negative. The staining patterns are illustrated in Figure 1.

Cytoplasmatic EGFR staining was also assessed in this study. Almost all tumor specimens stained uniformly with a weak cytoplasmatic intensity in more than $50 \%$ of the tumor cells. Expression of membranous EGFR showed no correlation with other parameters, including age, FIGO stage, tumor size, histology, pretreatment hemoglobin or COX-2 expression, which was also inde- pendent of histopathology and clinical characteristics. Table 1 also shows that $18 \%$ of the patients were classified as positive with respect to COX-2 expression meaning that more than $10 \%$ of the tumor cells stained with a moderate or strong intensity.

Of the 91 patients included in this study, 61 (67\%) were deceased by the time of this analysis. The median survival time for all included patients was 6.7 years [3.7 8.5 years] 95\% CI, with a range of 0.3 to 14.3 years.

The overall survival was significantly worse ( $\mathrm{p}=$ 0.0002) for patients with tumors staining positive for EGFR. The survival curve is shown in Figure 2(a). The median survival was 8.5 years (95\% CI, 5.9 - 10.5 years) for tumors with negative EGFR immunostaining, whereas the median survival for patients with EGFR positive tumors was 2.4 years (95\% CI, 1.3 - 3.6 years). Similarly, COX-2 overexpression was also associated with poor survival compared to patients without COX-2 overexpression $(\mathrm{P}=0.0084$, Figure 2(b)). The median survival time for the 75 patients with COX-2 negative tumors was 7.4 years (95\% CI, 5.5 - 10.2 years), while that of the 16 patients with COX-2 positive tumors was 2.3 years (95\% CI, 1.2 - 3.8 years). Since both EGFR and COX-2 expression were associated with overall survival, we evaluated the prognostic significance of these two markers in combination. Only 4 patients co-overexpressed both markers and an additional 28 patients overexpressed one of the markers, while the remaining 59 patients showed normal levels of both. Figure 2(c) illustrates the Kaplan-Meier curve for the combination of the two markers and it appears from the curve that patients with no overexpression of the two markers had significantly better survival $\left(\mathrm{P}<10^{-5}\right)$ compared to patients that were positive for either of the two markers. The median survival time was 10.2 years (95\% CI, 7.1 - 12.1 years) for patients negative for the two markers and 2.4 years (95\% CI, 1.5 - 3.6 years) for patients positive for one or two markers.

In a multivariate survival analysis both EGFR and COX-2 proved to be independent prognostic factors with hazard ratios of 2.43 and 1.94, respectively, with a longer survival for patients that showed no overexpression of the markers (Table 2(a)).

When analyzing the combination of EGFR and COX-2 in a multivariate COX regression model, also this combination of patients with overexpression of either both markers or just one of the markers proved to be an independent prognostic factor with a hazard ratio of 3.05 compared to patients that had no overexpression of either marker (Table 2(b)).

\section{Discussion}

The literature dealing with prognostic markers in cervix 


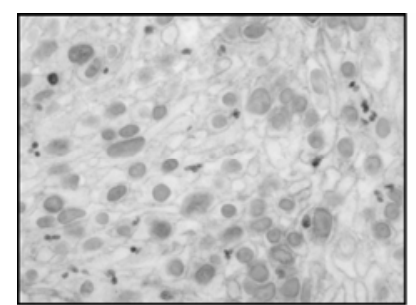

(a)

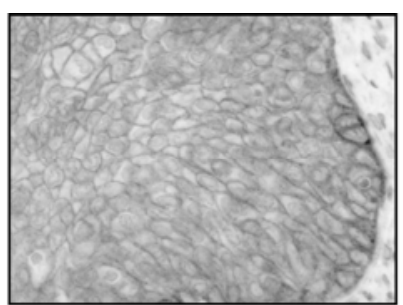

(b)

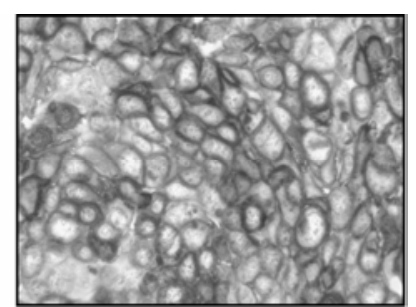

(c)

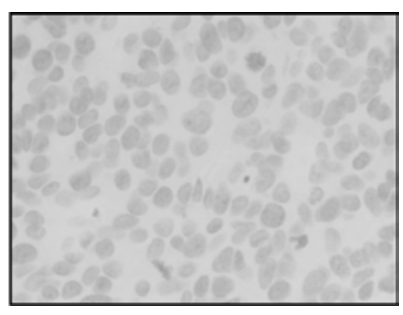

(d)

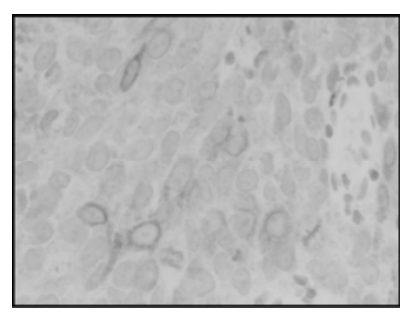

(e)

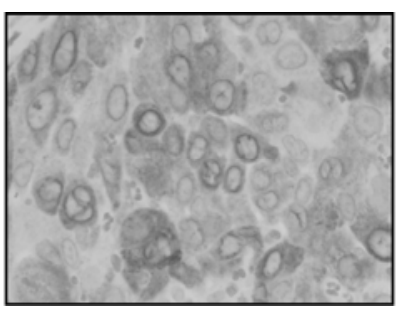

(f)

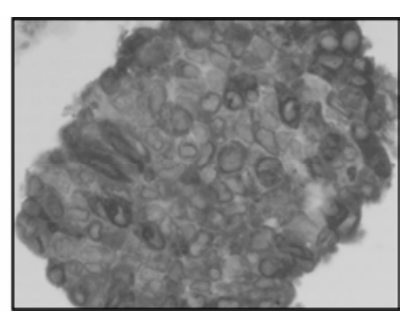

(g)

Figure 1. Different immunohistochemic stainings of EGFR and COX-2. (a) EGFR, low; (b) EGFR, moderate; (c) EGFR, strong; (d) COX-2, negative; (e) COX-2, low; (f) COX-2 moderate; (g) COX-2, strong.

Table 2. Multivariate survival analysis.

(a)

\begin{tabular}{ccccccc}
\hline Clinicopathological characteristics & B regression coefficient & SE (B) & HR & 95\% CI (HR) & $P$ value \\
\hline Age & 0.007 & 0.012 & 1.01 & $0.98-1.03$ & 0.561 \\
FIGO stage & 0.350 & 0.140 & 1.42 & $1.08-1.87$ & 0.013 \\
EGFR & 0.887 & 0.294 & 2.43 & $1.36-4.33$ & 0.003 \\
COX-2 & 0.662 & 0.316 & 1.94 & $1.04-3.60$ & 0.037 \\
\hline
\end{tabular}

(b)

\begin{tabular}{ccccccc}
\hline Clinicopathological characteristics & B regression coefficient & SE (B) & HR & 95\% CI (HR) & $P$ value \\
\hline Age & 0.010 & 0.012 & 1.01 & $0.99-1.03$ & 0.391 \\
FIGO stage & 0.386 & 0.139 & 1.47 & $1.12-1.93$ & 0.005 \\
Combined EGFR/COX-2 & 1.115 & 0.267 & 3.05 & $1.81-5.14$ & $<0.00001$ \\
\hline
\end{tabular}

cancer is broad [3,5-14]. Several traditional histopathological markers, e.g. grade, have been claimed to hold prognostic information, but none of the different grading systems have been generally accepted for daily routine use [10]. The issue is important, as more aggressive treatment with more toxicity should be based on a rational selection of patients.

A number of publications have suggested that the expression of EGFR may serve as a prognostic marker. Kersemaekers [6] included 136 stage I and II patients and observed a moderate/strong expression in 54\% of the tumors. EGFR overexpression was associated with a worse prognosis as estimated by disease free $(p=0.002)$ and overall ( $\mathrm{p}=0.003$ ) survival. The independent prognostic importance was verified in a multivariate analysis. Kristensen et al. analyzed 132 patients stage Ib. EGFR overexpression was found in $25.8 \%$ of the tumors with a clear negative prognostic impact [7]. The results are confirmed in advanced cervix cancer by our findings although our rate of overexpression was lower (22\%), a fact that may be explained by different methods and probably also different evaluation of the immunohistochemic stainings.

Kim et al. [3] included all stages in a study of 73 patients and reported an independent prognostic importance of EGFR expression using an enzyme immuno-assay. It 


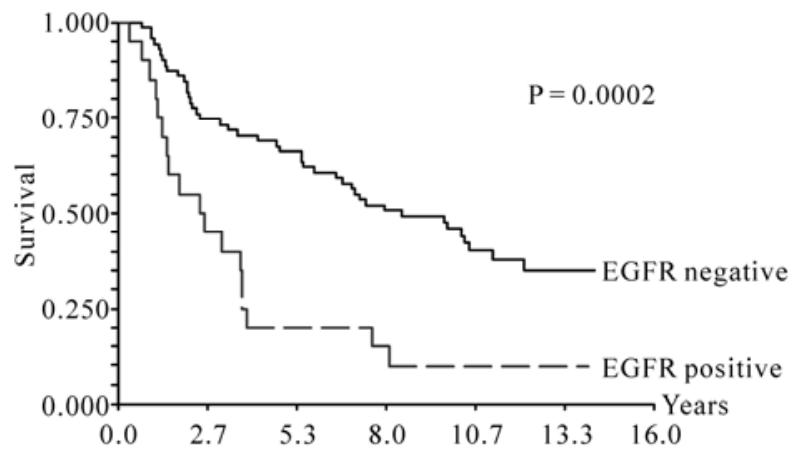

(a)

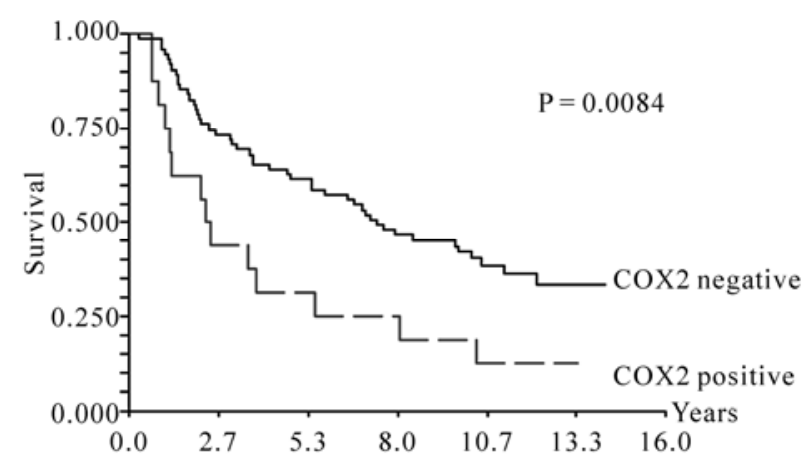

(b)

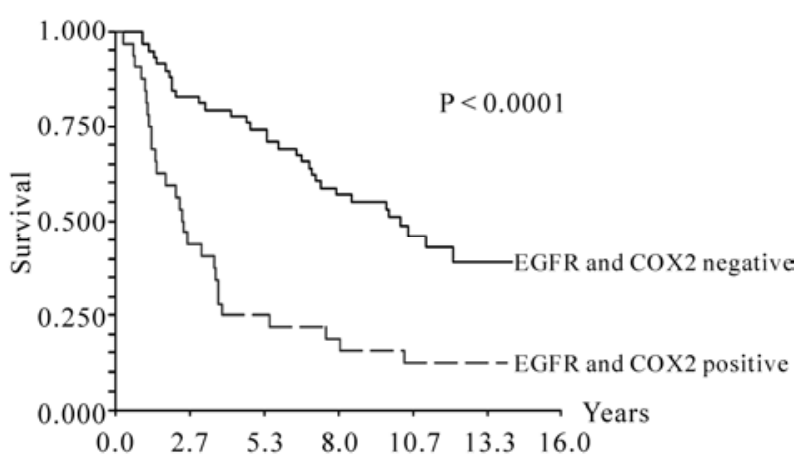

(c)

Figure 2. (a) Overall survival according to EGFR expression; (b) Overall survival according to COX-2 expression; (c) Overall survival according to combined EGFR and COX-2 expression.

should, however, be mentioned that negative results also have been reported. Scambia et al. [11] found no relationship between EGFR expression and overall survival. It should be noticed that their approach was a quantitative measurement of the EGFR protein, which cannot without reservations be compared to immunohistochemistry.

Furthermore, it is important to realize that immunohistochemistry is not an objective method and it is only semi-quantitative at best. Consequently, diverging results are more or less bound to occur. There is evidence that
EGFR overexpression occurs more often in the squamous cell carcinoma compared to the other types [8,12]. When comparing different results, the tumor types should be accounted for.

Lindström et al. [12] included 129 women with squamous cell carcinomas and compared them with 31 women with adenocarcinomas with respect to expression of 11 different tumor markers including EGFR and COX-2. It was found that EGFR in fact was significantly over-expressed in squamous cell carcinomas of the cervix compared to adenocarcinomas. The study concluded that COX-2 is associated with poor prognostic outcome for women with squamous cell carcinomas. EGFR on the other hand was not found to correlate to survival in either histological type. Both tumor markers were found to be more highly expressed in adenocarcinomas compared to squamous cell carcinomas. The results suggest that future studies should be conducted on separate histological subtypes. In the present study we were not able to examine this difference because of the small sample of adenocarcinomas.

Overexpression of COX-2 can be found in inflammation but also in the pre-invasive stage of cervix lesions. The overexpression in cervix cancer seems to be associated with advanced stages and distant metastases [13]. In stage Ib COX-2 expression failed to demonstrate a prognostic significance although it had a significant correlation with lymph node metastases [9]. COX-2 holds prognostic information when over-expressed in stage IIIb-IVa cervical cancer as also seen in the present study [5]. The combination of EGFR and COX-2 seems to hold additive prognostic information as shown by Kim et al. [14]. They divided their patients into four groups according to EGFR and COX-2 expression. The patients with positivity for both markers had a poor prognosis compared to the other three groups. It should, however, by noticed that some of the groups were very small (8 patients) and the results should be interpreted with caution. Our result indicates that an overexpression of one of the markers signalizes a poor prognosis, which holds true in a multivariate analysis.

Over-expression of COX-2 may have a radio-protective effect [15]. It is therefore obvious to use COX-2 inhibitors as radiosensitizers and there are in vitro studies indicating that e.g. celecoxib has an oxygen enhance factor of 1.9 [16]. However, clinical studies have been disappointing. In a phase II trial Herera et al. [17] found a high rate $(35.5 \%)$ of grade 3 and 4 toxicity, but no difference in response rate. Similar results were reported in a radiation therapy oncology group (RTOG) study [18] with 128 patients. Grade 3 and 4 toxicity was reported in $47 \%$ of the patients, but there was no significant increase in efficiency. A phase II trial in rectal cancer was also 
negative [19]. The possible clinical importance of COX-2 in cervix cancer needs further clarification.

Several studies have been conducted regarding the clinical and therapeutic importance of EGFR over-expression $[6,7,11,12,20-22]$. It is widely known that one of the challenges in chemotherapeutic treatment is drug resistance. Promising results are shown in in vitro studies using cetuximab, a monoclonal antibody targeting the EGFR, thus exploiting the fact that EGFR is over-expressed in cervical cancer cells. EGF and cetuximab compete for the binding site on EGFR and after binding it causes internalization without activation of the tyrosine kinase activity causing downmodulation of EGFR consequently inhibiting proliferation [20-22]. Furthermore, in vitro studies have indicated an EGFR level-dependent increase of resistance to radiotherapy in ovarian cell lines, which is converted by treatment with a monoclonal antibody that downregulates EGFR [23]. This might also be the case for cervical cancer cells, but further studies are needed to clarify the matter.

In conclusion the present study shows that EGFR and COX-2 are important prognostic markers in stage IIb-IVa cervix cancer. The results need verification in prospective trials.

\section{Declaration of Interest}

The authors report no conflicts of interest. The authors alone are responsible for the content and writing of the paper.

\section{REFERENCES}

[1] C. Vale, J. F. Tierney, L. A. Stewart, M. Brady, K. Dinshaw, A. Jakobsen, et al., "Reducing Uncertainties about the Effects of Chemoradiotherapy for Cervical Cancer: A Systematic Review and Meta-Analysis of Individual Patient Data from 18 Randomized Trials,” Journal Clinical Oncology, Vol. 26, No. 35, December 2008, pp. 58025812. doi:10.1200/JCO.2008.16.4368

[2] W. J. Gullick, "Prevalence of Aberrant Expression of the Epidermal Growth Factor Receptor in Human Cancers," British Medical Bulletin, Vol. 1, No. 47, January 1991, pp. 87-98.

[3] J. W. Kim, Y. T. Kim, D. K. Kim, C. H. Song and J. W. Lee, "Expression of Epidermal Growth Factor Receptor in Carcinoma of the Cervix," Gynecologic Oncology, Vol. 2, No. 60, February 1996, pp. 283-287. doi:10.1006/gyno.1996.0039

[4] W. R. Hanson and C. Thomas, “16, 16-Dimethyl ProsTaglandin E2 Increases Survival of Murine Intestinal Stem Cells When Given before Photon Radiation," $R a$ diat Research, Vol. 2, No. 96, November 1983, pp. 393-398. doi:10.2307/3576222

[5] J. L. Young, A. A. Jazaeri, C. J. Darus and S. C. Modesitt, "Cyclooxygenase-2 in Cervical Neoplasia: A Review,"
Gynecologic Oncology, Vol. 1, No. 109, April 2008, pp. 140-145. doi:10.1016/j.ygyno.2008.01.008

[6] A. M. Kersemaekers, G. J. Fleuren, G. G. Kenter, L. J. Van den Broek, S. M. Uljee, J. Hermans, et al., "Oncogene Alterations in Carcinomas of the Uterine Cervix: Overexpression of the Epidermal Growth Factor Receptor is Associated with Poor Prognosis,” Clinical Cancer Research, Vol. 3, No. 5, March 1999, pp. 577-586.

[7] G. B. Kristensen, R. Holm, V. M. Abeler and C. G. Trope, "Evaluation of the Prognostic Significance of Cathepsin D, Epidermal Growth Factor Receptor and c-erbB-2 in Early Cervical Squamous Cell Carcinoma. An ImmunoHistochemical Study," Cancer, Vol. 3, No. 78, August 1996, pp. 433-440. doi:10.1002/(SICI)1097-0142(19960801)78:3<433::AIDCNCR9>3.0.CO;2-K

[8] F. Baltazar, A. L. Filho, C. Pinheiro, M. A. Moreira, G. S. Queiroz, G. J. Oton, A. F. Júnior, L. F. Ribeiro and F. C. Schmitt, "Cyclooxygenase-2 and Epidermal Growth Factor Receptor Expressions in Different Histological Subtypes of Cervical Carcinomas," International Journal of Gynecologic Pathology, Vol. 3, No. 26, July 2007, pp. 235-241. doi:10.1097/pgp.0b013e31802f1996

[9] T. Manchana, S. Triratanachat, N. Sirisabya and A. Vasuratna, W. Termrungruanglert and D. Tresukosol, "Prevalence and Prognostic Significance of COX-2 Expression in Stage IB Cervical Cancer," Gynecologic Oncology, Vol. 3, No. 100, March 2006, pp. 556-560. doi:10.1016/j.ygyno.2005.09.014

[10] N. Singh and S. Arif, "Histopathologic Parameters of Prognosis in Cervical Cancer-A Review,” International Journal of Gynecologic Cancer, Vol. 5, No. 14, September-October 2004, pp. 741-750. doi:10.1111/j.1048-891X.2004.014504.X

[11] G. Scambia, G. Ferrandina, M. Distefano, G. D’Agostino, P. Edetti-Panic and S. Mancuso, "Epidermal Growth Factor Receptor (EGFR) is not Related to the Prognosis of Cervical Cancer,” Cancer Letter, Vol. 2, No. 123, January 1998, pp. 135-139. doi:10.1016/S0304-3835(97)00421-7

[12] A. K. Lindström, T. Tot, U. Stendahl, S. Syrjänen, K. Syrjänen and D. Hellberg, "Discrepancies in Expression and Prognostic Value of Tumor Markers in Adenocarcinoma and Squamous Cell Carcinoma in Cervical Cancer," Anticancer Research, Vol. 7, No. 29, July 2009, pp. 2577- 2578.

[13] Y. B. Kim, G. E. Kim, N. H. Cho, H. R. Pyo, S. J. Shim, S. K. Chang, et al., "Overexpression of Cyclooxygenase-2 is Associated with a Poor Prognosis in Patients with Squamous Cell Carcinoma of the Uterine Cervix Treated with Radiation and Concurrent Chemotherapy," Cancer, Vol. 3, No. 95, 2002, pp. 531-539. doi:10.1002/cncr.10684

[14] G. E. Kim, Y. B. Kim, N. H. Cho, H. C. Chung, H. R. Pyo, J. D. Lee, et al., "Synchronous Coexpression of Epidermal Growth Factor Receptor and Cyclooxygenase-2 in Carcinomas of the Uterine Cervix: A Potential Predictor of Poor Survival,” Clinical Cancer Research, Vol. 4, No. 
10, February 2004, pp. 1366-1374. doi:10.1158/1078-0432.CCR-0497-03

[15] H. Ishikawa, T. Ohno, S. Kato, M. Wakatsuki, M. Iwakawa, T. Ohta et al., "Cyclooxygenase-2 Impairs Treatment Effects of Radiotherapy for Cervical Cancer by Inhibition of Radiation-Induced Apoptosis,” International Journal of Radiation Oncology Biology Physics, Vol. 3, No. 66, December 2006, pp. 1347-1355. doi:10.1016/j.ijrobp.2006.07.007

[16] T. W. Davis, N. Hunter, O. C. Trifan, L. Milas and J. L. Masferrer, "COX-2 Inhibitors as Radio Sensitizing Agents for Cancer Therapy," American Journal of Clinical Oncology, Vol. 4, No. 26, August 2003, pp. 58-61. doi:10.1097/01.COC.0000074158.59269.9F

[17] F. G. Herrera, P. Chan, C. Doll, M. Milosevic, A. Oza, A. Syed, et al., "A Prospective Phase I-II Trial of the Cyclooxygenase-2 Inhibitor Celecoxib in Patients with Carcinoma of the Cervix with Biomarker Assessment of the Tumor Microenvironment," International Journal of Radiation Oncology Biology Physics, Vol. 1, No. 67, January 2007, pp. 97-103. doi:10.1016/j.jjrobp.2006.08.024

[18] D. K. Gaffney, K. Winter, A. P. Dicker, B. Miller, P. J. Eifel, J. Ryu, et al., "A Phase II Study of Acute Toxicity for Celebrex (celecoxib) and Chemo Radiation in Patients with Locally Advanced Cervical Cancer: Primary Endpoint Analysis of RTOG 0128,” International Journal of Radiation Oncology Biology Physics, Vol. 1, No. 67, January 2007, pp. 104-109. doi:10.1016/j.ijrobp.2006.08.002

[19] A. Jakobsen, J. P. Mortensen, C. Bisgaard, J. Lindebjerg, S. R. Rafaelsen and V. O. Bendtsen, "A COX-2 Inhibitor Combined with Chemoradiation of Locally Advanced Rectal Cancer: A Phase II Trial,” International Journal of Gynecologic Pathology, Vol. 3, No. 23, March 2008, pp. 251-255. doi:10.1007/s00384-007-0407-7

[20] S. Bellone, G. Frera, G. Landolfi, C. Romani, E. Bandiera, G. Tognon, J. J. Roman, A. F. Burnett, S. Pecorelli and A. D. Santin, "Over Expression of Epidermal Growth Factor Type-1 Receptor (EGF-R1) in Cervical Cancer: Implications for Cetuximab-Mediated Therapy Inrecurrent/Metastatic Disease,” Gynecology Oncology, Vol. 3, No. 106, September 2007, pp. 513-520. doi:10.1016/j.ygyno.2007.04.028

[21] E. S. Kim, F. R. Khuri and R. S. Herbst, "Epidermal Growth Factor Receptor Biology (IMC-C225),” Current Opinion Oncology, Vol. 6, No. 13, November 2001, pp. 506-513. doi:10.1097/00001622-200111000-00014

[22] J. M. Del Campo, A. Prat, A. Gil-Moreno, J. Perez and M. Parera, "Update on Novel Therapeutic Agents for Cervical Cancer,” Gynecologic Oncology, Vol. 3 (Suppl. 2), No. 110, September 2008, pp. 72-76.

[23] K. Liang, K. K. Ang, L. Milas, N. Hunter and Z. Fan, "International Journal of Radiation Oncology Biology," Physics, Vol. 1, No. 57, 2003, pp. 246-254. doi:10.1016/S0360-3016(03)00511-X

\section{Abbreviations}

\author{
EGF Epidermal Growth Factor \\ EGFR Epidermal Growth Factor Receptor \\ VEGF Vascular Endothelial Growth Factor \\ COX-2 Cyclooxygenase-2 \\ RTOG Radiation Therapy Oncology Group
}

